\title{
Methylation analysis of 79 patients with growth restriction reveals novel patterns of methylation change at imprinted loci
}

\author{
Claire Louise Susan Turner ${ }^{1,2}$, Deborah M Mackay ${ }^{2,3}$, Jonathan LA Callaway ${ }^{2,3}$, Louise E Docherty ${ }^{2,3}$, \\ Rebecca L Poole ${ }^{2,3}$, Hilary Bullman ${ }^{2}$, Margaret Lever ${ }^{2}$, Bruce M Castle $^{2}$, Emma C Kivuva $^{4}$, \\ Peter D Turnpenny ${ }^{4}$, Sarju G Mehta ${ }^{5}$, Sahar Mansour ${ }^{6}$, Emma L Wakeling ${ }^{7}$, Verghese Mathew ${ }^{8}$, \\ Jackie Madden ${ }^{9}$, Justin H Davies ${ }^{10}$ and I Karen Temple ${ }^{\star, 1,2,3}$
}

This study was an investigation of 79 patients referred to the Wessex Regional Genetics Laboratory with suspected RussellSilver Syndrome or unexplained short stature/intra uterine growth restriction, warranting genetic investigation. Methylation status was analysed at target sequences within eleven imprinted loci (PLAGL1, IGF2R, PEG10, MEST1, GRB10, KCNQ10T1, H19, IGF2PO, DLK1, PEG3, NESPAS). Thirty seven percent (37\%) (29 of 79) of samples were shown to have a methylation abnormality. The commonest finding was a loss of methylation at $H 19$ (23 of 29), as previously reported in Russell-Silver Syndrome. In addition, four of these patients had methylation anomalies at other loci, of whom two showed hypomethylation of multiple imprinted loci, and two showed a complete gain of methylation at IGF2R. This latter finding was also present in five other patients who did not have demonstrable changes at $H 19$. In total, 7 of 79 patients showed a gain of methylation at IGF2R and this was significantly different from a normal control population of 267 individuals $(P=0.002)$. This study in patients with growth restriction shows the importance of widening the epigenetic investigation to include multiple imprinted loci and highlights potential involvement of the IGF2R locus.

European Journal of Human Genetics (2010) 18, 648-655; doi:10.1038/ejhg.2009.246; published online 27 January 2010

Keywords: growth restriction; Russell-Silver syndrome; 11 p15 epimutation; H19; IGF2R; hypomethylation of imprinted loci

\section{INTRODUCTION}

Imprinted genes are of fundamental importance in normal human growth and development. Their expression is restricted in a parent-oforigin specific manner by epigenetic modifications, including DNA methylation and histone modification. ${ }^{1}$

A number of imprinting syndromes due to aberrant expression of imprinted genes have been described. These include transient neonatal diabetes (TND), Russell-Silver syndrome (RSS), BeckwithWiedemann syndrome (BWS), Prader-Willi syndrome, Angelman syndrome, Pseudohypoparathyroidism type $1 \mathrm{~b}$, maternal (also called Temple syndrome ${ }^{2}$ ) and paternal UPD 14 related disorder. Though this list represents diverse phenotypes, there are common features: phenotypically, many are associated with disordered growth; molecularly, each may be caused by aberrant methylation at a differentially methylated region (DMR). In some of these conditions in addition to the site-specific loss of methylation (LOM) associated with the disorder, LOM also occurs at diverse additional imprinted loci. This has been termed hypomethylation of multiple imprinted loci (HIL) ${ }^{3}$ and has been observed in BWS and TND. ${ }^{3-5}$
RSS is characterised by pre- and post-natal growth restriction; other salient features include relative head sparing, a triangular face with prominence of the forehead, a low body mass index, asymmetry, and feeding difficulties in infancy. RSS remains a difficult diagnosis to make due to clinical and (epi)genetic heterogeneity. Various clinical criteria have been proposed ${ }^{6,7}$ but many of the features are relatively nonspecific, and there is considerable phenotypic overlap between RSS, and low birth weight or short stature due to other causes. Furthermore, some of the features become less apparent with increasing age. Molecularly, two well described causes exist: LOM at H19, which is found in 30-60\%, ${ }^{7-9}$ and maternal uniparental disomy of chromosome 7 (mUPD7), which is found in around $5 \%$ of well-defined cases. ${ }^{7}$ The critical region on chromosome 7 contributing to the RSS phenotype has not yet been established, and there are currently three candidate imprinted regions (MEST/PEG1, GRB10 and PEG10) at which an isolated epigenetic change may conceivably lead to an RSS phenotype.

Maternal duplications of the ICR2 region on $11 \mathrm{p} 15$ are also implicated in a small number of RSS cases. ${ }^{10-12}$ Thus, within RSS there are clear subgroups, which may have discrete clinical

\footnotetext{
${ }^{1}$ Academic Unit of Genetic Medicine, School of Medicine, University of Southampton, Princess Anne Hospital, Southampton, UK; ${ }^{2}$ Wessex Genetics Service, Southampton University Hospitals Trust, Southampton SO16 5YA and Salisbury Hospital NHS Foundation Trust, Salisbury, UK; ${ }^{3}$ Division of Human Genetics, University of Southampton,

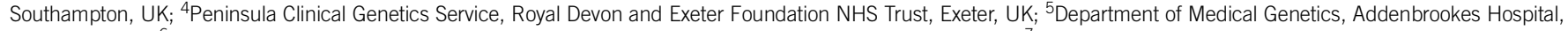
Cambridge, UK; ${ }^{6}$ SW Thames Regional Genetics Service, St George's, University of London, Tooting, London, UK; ${ }^{7} \mathrm{NW}$ Thames Regional Genetics Service, North West London Hospitals NHS Trust, Harrow, UK; ${ }^{8}$ Department of Paediatrics, Hull Royal Infirmary, Hull, UK; 9 Institute of Human Nutrition, University of Southampton, Southampton, UK; ${ }^{10}$ Department of Child Health, Southampton University Hospital NHS Trust, Southampton, UK

*Correspondence: Dr IK Temple, Wessex Clinical Genetics Service, Academic Unit of Genetic Medicine, School of Medicine, University of Southampton, Princess Anne Hospital, Coxford Road, Southampton SO16 5YA, UK. Tel: +44 238079 6170; Fax: +44 238079 4346; E-mail: IKT@southampton.ac.uk

Received 20 May 2009; revised 17 December 2009; accepted 22 December 2009; published online 27 January 2010
} 
implications. Furthermore, at least $30 \%$ of clinically diagnosed cases have no recognised cause.

In patients with RSS due to LOM at H19, HIL has not yet been observed; in mice LOM at $H 19$ can be accompanied by LOM at $D L K 1^{13}$ but a recent study examining methylation at four loci (PLAGL1, DLK1, MEST, H19) in RSS patients, found no additional LOM. ${ }^{14}$

The possibility that LOM at H19, or mUPD7 might not be specific to RSS and might be found in a proportion of patients with isolated pre- and postnatal growth retardation, has also been considered by others. ${ }^{7,8,15,16}$ Until recently, the conclusion in each of these studies was that mUPD7 and LOM at H19 were restricted to those with typical RSS features. More recently, less typical patients have been reported. ${ }^{17}$ Comprehensive methylation studies at other known DMRs have not yet been published in the cohort with atypical RSS.

We hypothesized that altered methylation patterns at the DMRs of imprinted genes on chromosome 7, or elsewhere, would be found in patients with growth restriction. Furthermore, given the observed HIL in some imprinting syndromes, we postulated that additional LOM would be seen in patients with LOM H19, defining a new subgroup of patients.

\section{MATERIALS AND METHODS}

DNA samples from 79 patients referred to the Wessex Regional Genetics Laboratory with growth restriction were included in the study. There were two recruitment groups: the first consisted of 19 patients recruited into the Imprinting Disorders Finding Out Why? (IDFOW) study. This group was referred with growth restriction, where the referring geneticist felt there was a potential imprinting disorder. Detailed phenotypic information accompanied these referrals. The second group comprised 60 samples sent to the NHS laboratory for the routine genetic investigation of unexplained short stature/intrauterine growth retardation, or possible SRS, as the major reason for referral. This latter group was identified by a single observer searching the laboratory database of referrals for the period 2000-2008. An appropriate referral reason and sufficient DNA were prerequisite selection criteria. These samples were anonymised and linked to clinical data. Twenty-seven of the 60 were referred by NHS consultants in Clinical Genetics from the United Kingdom. The remaining 33 samples originated from local paediatricians.

Each recruitment group had its own ethics approval: the first group was adopted by the UK comprehensive local research network, and approved by Southampton and South West Hampshire Research Ethics committee 07/H0502/85; the second group were approved by the Salisbury Research Ethics Committee REC05/Q2008/52, but the approval did not allow us to go back to the patients or referring clinicians for more detailed phenotypic information.

The two recruitment arms generated four main categories of referral groups: RSS; short stature (SS) sufficient to warrant genetic investigation; intrauterine growth retardation (IUGR); IUGR and unexplained short stature.

DNA was extracted from peripheral blood lymphocytes by standard procedures. No additional tissue samples were available for this study.

Methylation status was analysed at target sequences within 11 imprinted loci (PLAGL1, IGF2R, PEG10, MEST1, GRB10, KCNQ1OT1, H19, IGF2P0, DLK1, PEG3, NESPAS). Genomic DNA was modified by bisulphite treatment (EZ DNA Methylation Kit; Zymo Research). Methylation-specific PCR (MS-PCR) was performed at these loci as previously described. ${ }^{3}$ Previously undescribed MS-PCR primer sets are given in Table 1. Each experiment was performed in duplicate.

DNA methylation was calculated as the peak height ratio of unmethylated and methylated amplicons and normalised against normal controls ( $>4$ per experiment). The methylation ratio is therefore the normalised unmethylated/ methylated peak height ratio as exemplified in Figure 1. A complete gain of methylation is represented as ' 0 '. Hypomethylation, that is, greater peak intensity for the unmethylated than the methylated amplicon, is represented by numbers $>1$. Hypermethylation, that is, greater peak intensity for the methylated than the unmethylated amplicon, is represented by numbers $<1$.

A patient result was considered abnormal when it fell outside three SD from the control mean normalised ratio.

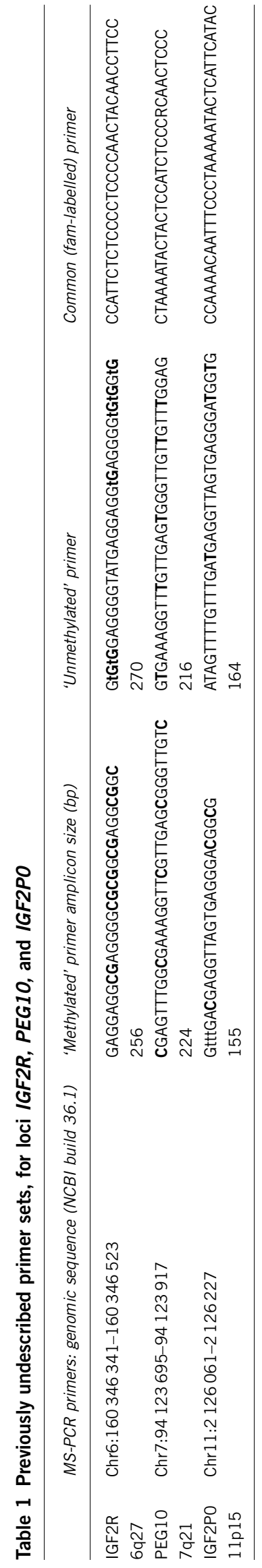




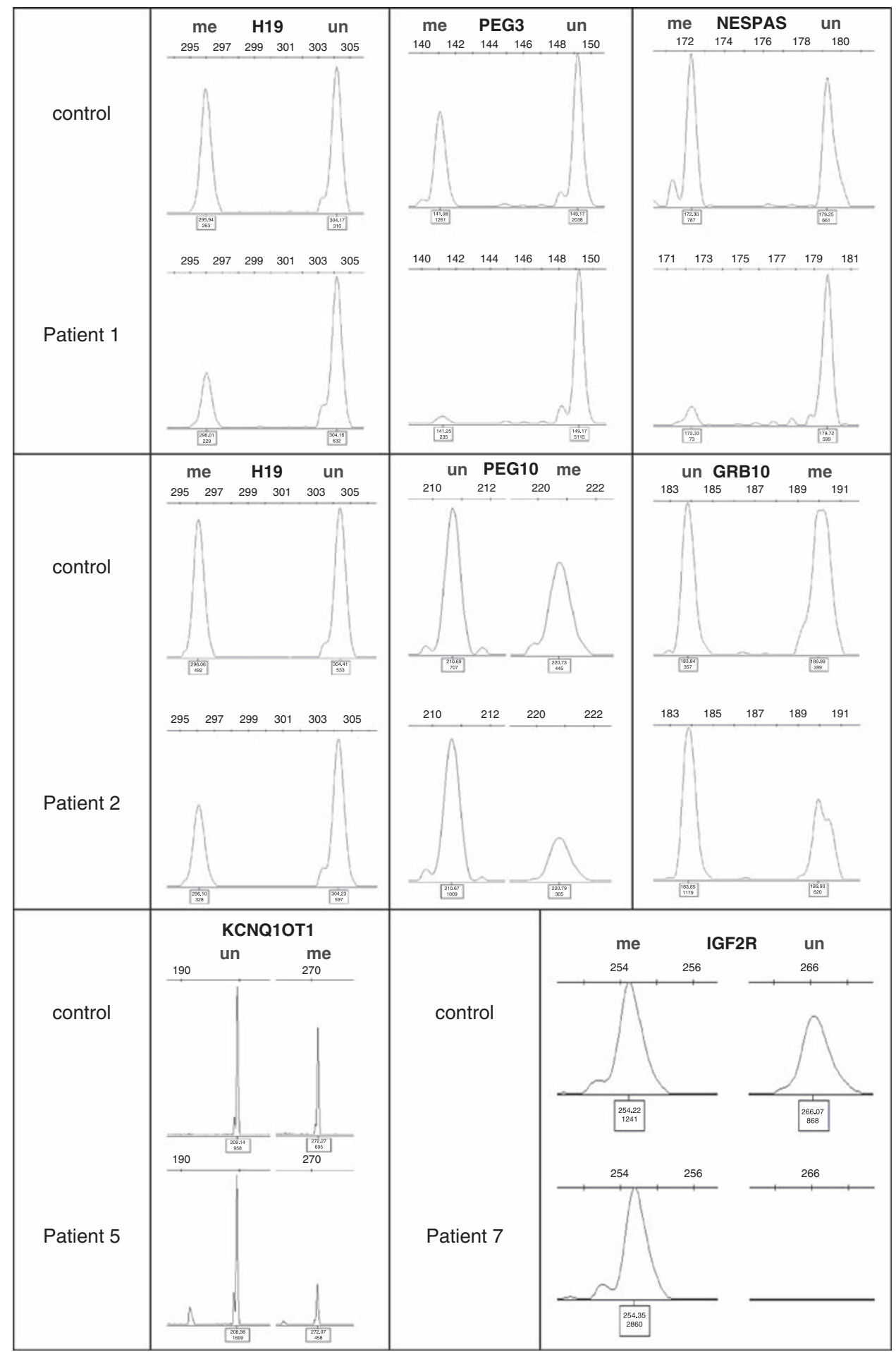

Figure 1 Electropherograms of methylation-specific PCR amplicons. Top row, patient 1; second row, patient 2; bottom row left, patient 5, bottom row right, patient 7. In each case an upper electropherogram represents the result from a control within the same experiment, and the lower electropherogram the result from the patient. The amplicon is identified above each panel, as are the methylated and unmethylated peaks (marked in red or blue for maternal or paternal origin, respectively). Presence of both peaks at equivalent abundance to the normal control is consistent with a normal methylation profile; reduction of the methylated peak height indicates relative hypomethylation at this site in a patient.

This method has been published elsewhere $e^{3,18,19}$ and has been used to detect methylation changes at several loci. To further substantiate the methods for each loci analysed within this paper, separate experiments were performed using control DNA derived from 126 normal controls (for IGF2R we used
267 controls). These experiments generated the SD of the ratios, providing further evidence of reproducibility (Table 3).

Abnormal results were confirmed using a separate bisulphited sample and also, in most cases, utilising a second methodology: for H19, IGF2R, GRB10, 
Table 2 Summary of results, recruitment method, and referral category for all patients

\begin{tabular}{|c|c|c|c|c|c|}
\hline \multirow[b]{2}{*}{ Ascertainment cohort } & \multirow[b]{2}{*}{ Clinical referral category } & \multirow{2}{*}{\multicolumn{2}{|c|}{$\begin{array}{l}\text { Number of patients with loss of } \\
\text { methylation at H19 DMR }\end{array}$}} & \multicolumn{2}{|c|}{ Number of patients who in addition to $\mathrm{H} 19$ result showed } \\
\hline & & & & $\begin{array}{c}\text { Methylation aberrations in at least } \\
\text { one other locus (see Table 3) }\end{array}$ & $\begin{array}{c}\text { Normal methylation at other loci } \\
\text { (data not shown) }\end{array}$ \\
\hline \multirow[t]{6}{*}{ IDFOW $(n=19)$} & RSS $(n=6)$ & LOM & 5 & 2 & 3 \\
\hline & & Normal & 1 & 0 & 1 \\
\hline & IUGR and short stature $(n=9)$ & LOM & 0 & NA & NA \\
\hline & & Normal & 9 & 4 & 5 \\
\hline & Short stature $(n=4)$ & LOM & 0 & NA & NA \\
\hline & & Normal & 4 & 1 & 3 \\
\hline \multirow[t]{8}{*}{ Anonymised $(n=60)$} & $\operatorname{RSS}^{a}(n=28)$ & LOM & 16 & 2 & 14 \\
\hline & & Normal & 12 & 0 & 12 \\
\hline & IUGR and short stature ${ }^{a}(n=3)$ & LOM & 1 & 0 & 1 \\
\hline & & Normal & 2 & 1 & 1 \\
\hline & Short stature $^{a}(n=7)$ & LOM & 0 & NA & NA \\
\hline & & Normal & 7 & 0 & 7 \\
\hline & $\operatorname{IUGR}^{\mathrm{a}}(n=22)$ & LOM & 1 & 0 & 1 \\
\hline & & Normal & 21 & 0 & 21 \\
\hline
\end{tabular}

IDFOW, patients recruited through the Imprinting Disorders Finding Out Why study in which detailed phenotypic information accompanied samples; RSS, Russell-Silver syndrome; IUGR, intra-uterine growth retardation; LOM, loss of methylation; NA, not applicable.

aThe clinical referral categories for those individuals recruited as the anonymised cohort were derived from details given on referral cards, accompanying samples. Due to the potential inaccuracy in this phenotypic information, there is the possibility that an individual may have been inaccurately assigned to a given clinical category.

PEG3 and NESPAS, pyrosequencing was performed, using standard methodo$\operatorname{logy}^{3}$ and with primers designed to be non overlapping with MS-PCR primer sequences; for KCNQ1OT1 (and H19) an MLPA kit was used (obtained from MRC Holland http://www.mlpa.com).

\section{RESULTS}

The results of all 79 patients, their recruitment method and referral category are tabulated in Table 2. The MS-PCR results of patients with novel imprinting signatures are given in Table 3, and depicted in Figure 1. Examples of corroboratory pyrosequencing assays are depicted in Figure 2.

Of the 79 patients included in this study, 23 patients had LOM at H19, and 21 of these were referred with a clinical suspicion of RSS. The remaining two patients were referred with variable phenotypes and were recruited from the anonymised arm: one had IUGR and suffered from numerous chest infections; the other was a twin with IUGR, exomphalos, hypospadias, a cleft palate and a cardiac defect.

Of the 23 patients with LOM at $H 19$ there were two with additional widespread LOM (Table 3). Of these, patient 1 had LOM involving NESPAS/GNAS and PEG3. This patient was born with a cleft lip and palate, and had a birth weight below the 0.4 th centile. By 7 years of age, without the use of growth hormone, she had shown catch up growth with her height on the 25th centile. She was reported to have speech and language delay. This patient had a half sibling with BWS, and there was a family history of recurrent pregnancy losses (manuscript in preparation). The other patient (patient 2) had LOM at IGF2R, KCNQ1OT1, PEG10, GRB10 and NESPAS. This patient was one of monochorionic monoamniotic female twins with discordant growth. She was born at 31 weeks, with her birth weight on the 9th centile. She developed renal failure in the neonatal period due to renal dysplasia. Her height and weight at age 4 years were on the 2-9th centiles, with her head circumference on the 50th centile. Asymmetry was noted, and motor and speech milestones were met later than in her twin. DNA was not available from her twin.
Patients 3 and 4 had, in addition to LOM at H19, a complete gain of methylation (GOM) at IGF2R. Their clinical features are summarised in Table 4.

Of the 56 patients with no LOM at H19, one patient (patient 5) showed LOM at KCNQ1OT1 (chr11p15, DMR2), ordinarily associated with BWS. MLPA analysis of the $11 \mathrm{p} 15$ region did not reveal any copy number changes to account for the LOM at KCNQ1OT1. This female patient was born at 38 weeks gestation and weighed $2.03 \mathrm{Kg}$ (0.4th centile). Her mother had been diagnosed with polycystic ovarian syndrome, and the pregnancy was complicated by pre-eclampsia (from 34 weeks). In the neonatal period she developed hypoglycaemia and feeding problems with a poor suck. At 7 years of age she had height and head circumference $<0.4$ th centile, with her weight on the 3rd centile. She showed developmental delay, particularly affecting her speech and language.

In five patients (patients 6-10) the only epigenetic anomaly found was complete GOM at IGF2R. The known clinical features within this group are shown in Table 4. The incidence of GOM in our study population (7 of 79) was significantly higher than in an anonymised control population of unknown anthropometry (3 of 267: $P=0.002$ Fisher Exact).

\section{DISCUSSION}

In this cohort of patients referred for investigation of RSS/unexplained growth restriction, 37\% (29 of 79) of DNA samples showed LOM at one or more imprinted loci. LOM at H19 was the most common methylation anomaly in such patients. Most other studies have concluded that LOM at $\mathrm{H} 19$ is very unlikely in those patients without features of RSS; $7,8,15,16$ however, in this series two patients with LOM at $H 19$ did not have a diagnosis of RSS clearly suggested on the referral details. They were both from the anonymised recruitment arm and therefore detailed phenotypic information has not been confirmed, but both were referred by clinical geneticists. In one of these patients there was a complex clinical picture and multiple congenital 


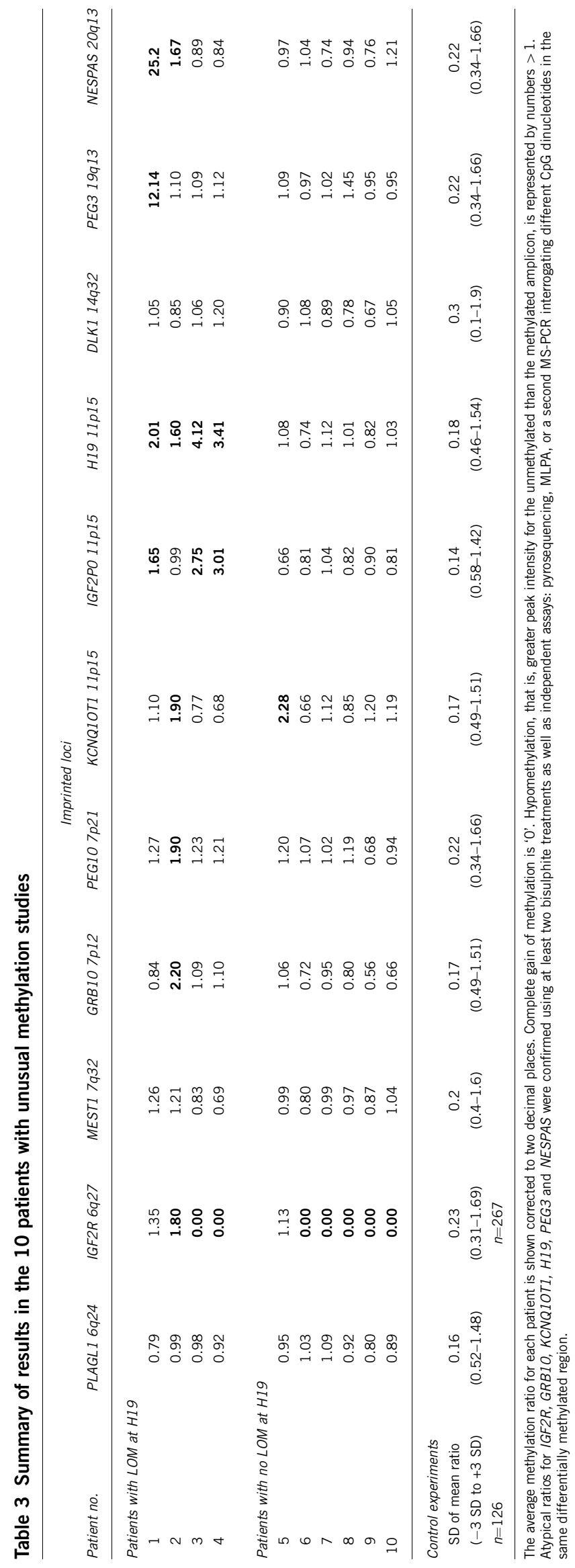

anomalies. It is possible that RSS had not been suspected clinically, because of the severity of comorbid features, or his young age.

We found HIL in 2 of 23 patients with LOM at H19, a phenomenon which was not observed in a previous study that tested a smaller number of DMRs. ${ }^{14}$ These two patients are remarkable. Patient 1 had a half-sibling with BWS. Siblings with RSS and BWS due to underlying HIL have not been previously described, and this family probably represents a new genetic disorder. Patient 2 was one of discordant monozygous twins, which might be aetiologically relevant: the incidence of monozygotic twinning in BWS is increased ${ }^{20,21}$ and some of these show HIL. ${ }^{22}$ Monozygous twins discordant for RSS have also been reported $;^{23-25}$ although, to our knowledge this latter group has not been investigated for HIL. We believe the discovery of HIL in patient 2 with growth restriction may represent a new aetiological group, with HIL arising as part of the twinning process.

With the exception of patient 2, who had LOM at GRB10 and PEG10 on chromosome 7 as part of a wider HIL, we did not detect epigenetic changes on chromosome 7 to account for the restricted growth in this cohort. These results corroborate those of another study that identified no methylation change at MEST/PEG1 in 54 RSS patients. ${ }^{26}$ Taken with our own results this suggests that isolated imprinting abnormalities of MEST1/PEG1, GRB10 and PEG10 on chromosome 7 are not a common cause of growth retardation or RSS.

Surprisingly, this study has also revealed that LOM at KCNQ1OT1, previously associated with BWS, can be found in patients with growth restriction (patients 2 and 5). Recently there has been another report of LOM at KCNQ1OT1 in three children without clinical BWS. ${ }^{27}$ In our study, although MLPA did not reveal a copy number change in the 11 p15 region, we cannot exclude an atypical paternal duplication, or a maternal deletion; however, it is difficult to explain the phenotype of growth restriction in the context of the epigenetic result in these two patients. In patient 2, the HIL involving multiple loci, including H19, may have prevented a net overgrowth; yet, a similar pattern, with hypomethylation at both maternally and paternally methylated loci, was also seen in discordant twins with BWS. ${ }^{22}$ The modest changes in methylation at multiple loci in patient 2 may reflect a dilutional effect resulting from circulation sharing with her twin. For patient 5, we did not show HIL, though we cannot exclude hypomethylation of DMRs not analysed in this study.

The finding of a complete gain of methylation at IGF2R in 7 of 79 patients is also intriguing. This occurred significantly more commonly in the patients (cases) than in our control group $(P=0.002$ using Fisher's Exact test).

Although the proliferative effects of IGF2 are mediated through the IGF1 receptor (IGF1R), the main function of the IGF2 receptor is anti-proliferative, achieved by clearing IGF2 from the circulation. ${ }^{28}$ Not surprisingly therefore, Igf $2 r$ knockout mice are up to $30 \%$ larger than wild-type mice. ${ }^{29-31}$ The expression of human and mouse IGF2R differs: in mice, $I g f 2 r$ imprinted expression is observed in all fetal and adult tissues; in humans, IGF2R imprinted expression has not been found in adult tissues, but has been found in fetal tissues and Wilms' tumours, in a proportion of samples tested. ${ }^{32}$ There is also a difference in the DMRs between these two species: DMR1, in the promoter of the Igf2r gene, occurs only in mice; DMR2 occurs in both species and is located in intron 2, methylated on the maternal allele. In mice the DMR2 acts as a promoter for Air, a non-coding RNA, which is antisense to $\operatorname{Igf2r}$. Maternal methylation of DMR2 silences maternal Air, allowing monoallelic maternal expression of IGF2R. There is now some evidence of a human Air homologue. ${ }^{33}$ Given this control and expression pattern, we hypothesised that a gain of methylation at DMR2 in humans might increase the expression of IGF2R (at a critical 


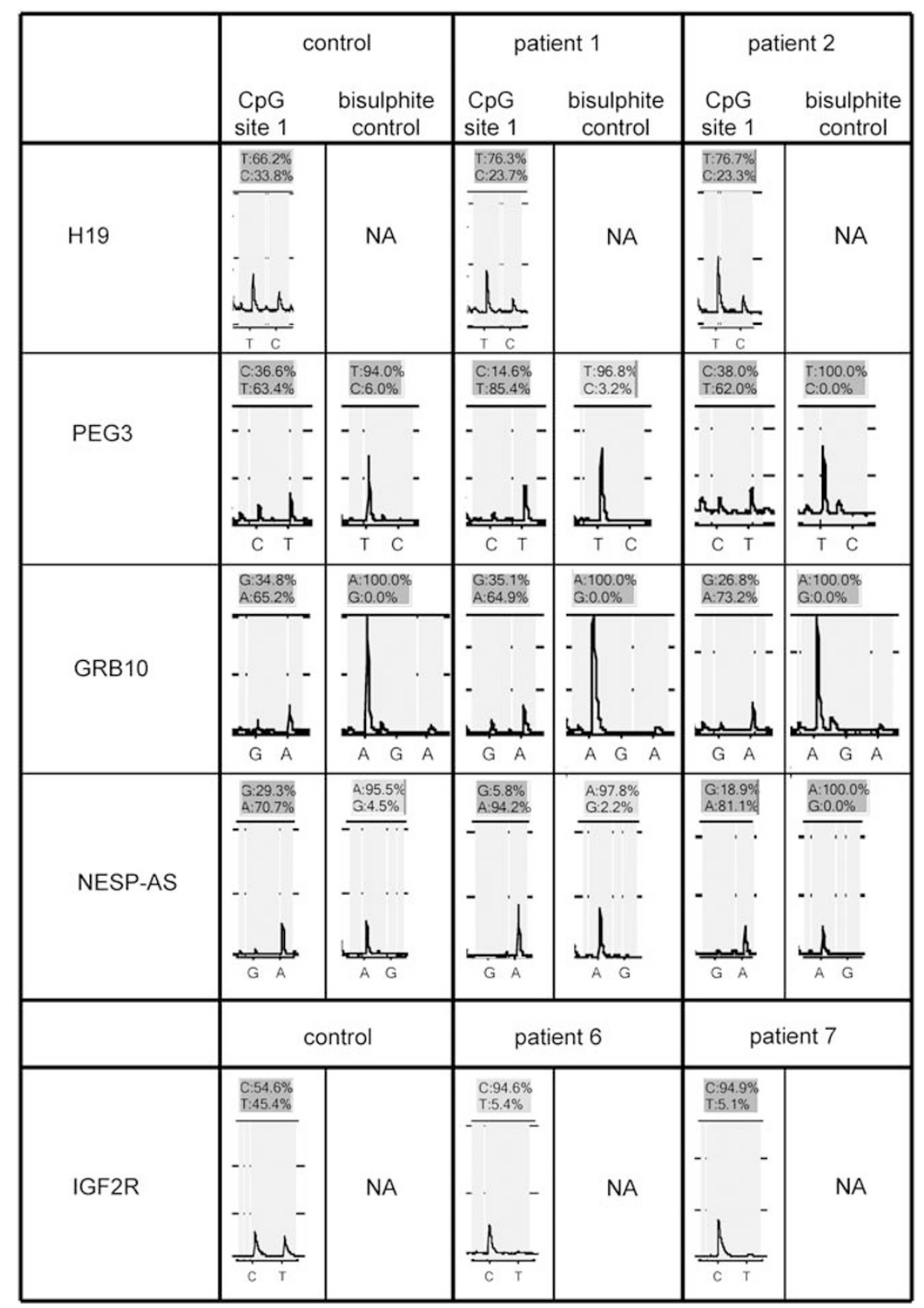

Figure 2 Results of allele quantification of bisulfite-induced C/T polymorphisms by pyrosequencing; examples of pyrograms. Successive columns illustrate a normal control, and patients. Successive rows illustrate the H19, PEG3, GRB10, NESPAS and IGF2R DMRs. Each table cell contains two images: on the left, the first $\mathrm{C} / \mathrm{T}$ polymorphism of the pyrosequencing reaction, and on the right, a bisulfite conversion control from the same pyrosequencing reaction (a $\mathrm{C}$ nucleotide not within a CpG dinucleotide, which would be expected to undergo 100\% C>T conversion). NA: Not available; for some assays no suitable cytosine was available to act as a conversion control. Figures above each image indicate the proportions of methylated and unmethylated product present at that position; alleles measured at $5 \%$ or less of total are indistinguishable from zero, owing to imprecise quantification at this level.

stage of embryonic development), which could lead to a decrease in circulating IGF2, and so decreased growth. The opposite epigenetic modification, in the form of demethylation of the DMR2 of Igf2r, leads to overgrowth of sheep. ${ }^{34}$ Furthermore, in humans a partial demethylation of DMR2 was detected in one of 55 patients with idiopathic overgrowth, and was associated with a decreased serum IGF2R level and decreased IGF2 binding, at age 3 years. ${ }^{35}$ We are not able to go back to the patients in this study to compare clinical findings in this subgroup with the rest of the cohort, or test IGF2 levels. The available clinical details reveal failure to thrive and post natal growth retardation, with or without prenatal growth retardation
(Table 4). The findings at IGF2R warrant further investigation and may have highlighted a novel subset of growth retarded individuals.

The main limitation of this study is the minimal phenotypic information available on the 60 individuals within the anonymised cohort. It is recognised that referral cards can be difficult to interpret and this may limit our epigenotype-phenotype correlations; however, almost half of this group originated from Clinical Geneticists who may provide more reliable referral details. Furthermore, this group does reflect routine clinical practice, being drawn from unselected referrals to a service laboratory of patients with growth restriction. Thus, this study mirrors the real difficulty of diagnosing RSS in the clinic: in 
Table 4 The phenotypic features of patients with GOM at IGF2R

\begin{tabular}{|c|c|c|c|}
\hline Patient & $\begin{array}{l}\text { Methylation status } \\
\text { at H19 }\end{array}$ & $\begin{array}{l}\text { Birth weight } \\
\text { centile }\end{array}$ & Other features \\
\hline 3 & LOM & Not known & Not known. \\
\hline 4 & LOM & Not known & Asymmetry, mild developmental delay, ventricular septal defect. \\
\hline 6 & Normal & 2nd & $\begin{array}{l}\text { Growth failure from } 3 \text { months. Age 19/12 <0.4th centile for height; OFC } 2 \text { nd centile. Normal development, } \\
\text { mild concern regarding speech. }\end{array}$ \\
\hline 7 & Normal & $<0.4$ th & $\begin{array}{l}\text { Feeding difficulties, micrognathia. Delayed dental eruption, vitiligo and anterior chamber dysgenesis. Age } 10 \text { years, } \\
\text { height remains }<0.4 \text { th centile. }\end{array}$ \\
\hline 8 & Normal & $\begin{array}{l}\text { Not known but } \\
\text { referred with IUGR }\end{array}$ & 0.4 th centile at time of referral. \\
\hline 9 & Normal & 50th & Developed unexplained short stature. \\
\hline 10 & Normal & $<0.4$ th & $\begin{array}{l}\text { Assisted conception; }<0.4 \text { th centile for weight at term and }<0.4 \text { th centile at } 3.5 \text { years; developmental } \\
\text { delay but minimal dysmorphic features. }\end{array}$ \\
\hline
\end{tabular}

many instances, although doctors know there is definite intrauterine growth retardation and or short stature, there maybe uncertainty about the presence of other RSS features, some of which evolve with time.

We believe this is the most extensive molecular study of its kind, analysing 11 DMRs in individuals with suspected RSS or growth retardation. We report new cases of HIL in patients with LOM at H19. We have ascertained further patients with LOM at KCNQ1OT1 who do not have clinical BWS. Although we did not identify the loci on chromosome 7 that may account for the growth restriction observed in maternal UPD7, we did identify a potentially important change involving IGF2R. We believe this study warrants confirmation prospectively alongside accurate clinical phenotyping

\section{ADDENDUM}

During the review process, Azzi et al ${ }^{36}$ also published evidence that some patients with RSS have hypomethylation involving multiple imprinted loci. Interestingly, they reported the involvement of different loci, which may indicate that more than one mechanism may be involved.

\section{CONFLICT OF INTEREST}

The authors declare no conflict of interest.

\section{ACKNOWLEDGEMENTS}

We thank the referring physicians and families for their participation. The study was funded by a Fellowship grant from Ipsen, awarded to CLS Turner.

1 Reik W, Walter J: Genomic imprinting: parental influence on the genome. Nat Rev Genet 2001; 2: 21-32.

2 Buiting K, Kanber D, Martin-Subero JI et al: Clinical features of maternal uniparental disomy 14 in patients with an epimutation and a deletion of the imprinted DLK1/GTL2 gene cluster. Hum Mutat 2008; 29: 1141-1146.

3 Mackay DJ, Callaway JL, Marks SM et al: Hypomethylation of multiple imprinted loci in individuals with transient neonatal diabetes is associated with mutations in ZFP57. Nat Genet 2008; 40: 949-951.

4 Bliek J, Verde G, Callaway J et al: Hypomethylation at multiple maternally methylated imprinted regions including PLAGL1 and GNAS loci in Beckwith-Wiedemann syndrome. Eur J Hum Genet 2008; 17: 611-619.

5 Rossignol S, Steunou V, Chalas C et al: The epigenetic imprinting defect of patients with Beckwith-Wiedemann syndrome born after assisted reproductive technology is not restricted to the 11 p15 region. J Med Genet 2006; 43: 902-907.

6 Price SM, Stanhope R, Garrett C, Preece MA, Trembath RC: The spectrum of SilverRussell syndrome: a clinical and molecular genetic study and new diagnostic criteria. J Med Genet 1999; 36: 837-842.

7 Netchine I, Rossignol S, Dufourg MN et al: $11 \mathrm{p} 15$ imprinting center region 1 loss of methylation is a common and specific cause of typical Russell-Silver syndrome: clinical scoring system and epigenetic-phenotypic correlations. J Clin Endocrinol Metab 2007; 92: 3148-3154.

8 Bartholdi D, Krajewska-Walasek M, Ounap $\mathrm{K}$ et al: Epigenetic mutations of the imprinted IGF2-H19 domain in Silver-Russell syndrome (SRS): results from a large cohort of patients with SRS and SRS-like phenotypes. J Med Genet 2009; 46: 192-197.

9 Bruce S, Hannula-Jouppi K, Peltonen J, Kere J, Lipsanen-Nyman M: Clinically distinct epigenetic subgroups in Silver-Russell syndrome: the degree of $\mathrm{H} 19$ hypomethylation associates with phenotype severity and genital and skeletal anomalies. J Clin Endocrinol Metab 2009; 94: 579-587.

10 Fisher AM, Thomas NS, Cockwell A et al: Duplications of chromosome 11p15 of maternal origin result in a phenotype that includes growth retardation. Hum Genet 2002; 111: 290-296.

11 Eggermann T, Meyer E, Obermann C et al: Is maternal duplication of $11 \mathrm{p} 15$ associated with Silver-Russell syndrome? J Med Genet 2005; 42: e26.

12 Schonherr N, Meyer E, Roos A, Schmidt A, Wollmann HA, Eggermann T: The centromeric $11 \mathrm{p} 15$ imprinting centre is also involved in Silver-Russell syndrome. J Med Genet 2007; 44: 59-63.

13 Kato $\mathrm{Y}, \mathrm{Kaneda} \mathrm{M}$, Hata $\mathrm{K}$ et al: Role of the Dnmt3 family in de novo methylation of imprinted and repetitive sequences during male germ cell development in the mouse. Hum Mol Genet 2007; 16: 2272-2280.

14 Schonherr N, Meyer E, Binder G, Wollmann HA, Eggermann T: No evidence for additional imprinting defects in Silver-Russell syndrome patients with maternal uniparental disomy 7 or 11 p15 epimutation. J Pediatr Endocrinol Metab 2007; 20: 1329-1331.

15 Eggermann T, Meyer E, Caglayan AO, Dundar M, Schonherr N: ICR1 epimutations in Ilp15 are restricted to patients with Silver-Russell syndrome features. J Pediatr Endocrinol Metab 2008; 21: 59-62.

16 Schonherr N, Meyer E, Eggermann K, Ranke MB, Wollmann HA, Eggermann T: (Epi)mutations in 11 p15 significantly contribute to Silver-Russell syndrome: but are they generally involved in growth retardation? Eur J Med Genet 2006; 49: 414-418.

17 Eggermann T, Gonzalez D, Spengler S, Arslan-Kirchner M, Binder G, Schonherr N: Broad clinical spectrum in Silver-Russell syndrome and consequences for genetic testing in growth retardation. Pediatrics 2009; 123: e929-e931.

18 Meyer E, Lim D, Pasha S et al: Germline mutation in NLRP2 (NALP2) in a familial imprinting disorder (Beckwith-Wiedemann Syndrome). PLoS Genet 2009; 5: e1000423.

19 Lim D, Bowdin SC, Tee L et al: Clinical and molecular genetic features of BeckwithWiedemann syndrome associated with assisted reproductive technologies. Hum Reprod 2009; 24: 741-747.

20 Berry AC, Belton EM, Chantler C: Monozygotic twins discordant for WiedemannBeckwith syndrome and the implications for genetic counselling. J Med Genet 1980; 17: $136-138$

21 Weksberg R, Shuman C, Caluseriu O et al: Discordant KCNQ1OT1 imprinting in sets of monozygotic twins discordant for Beckwith-Wiedemann syndrome. Hum Mol Genet 2002; 11: 1317-1325.

22 Bliek J, Alders M, Maas S et al: Lessons from BWS twins: complex maternal and paternal hypomethylation and a common source of haematopoietic stem-cells. Eur J Hum Genet 2009; 17: 1625-1634.

23 Yamazawa K, Kagami M, Fukami M, Matsubara K, Ogata T: Monozygotic female twins discordant for Silver-Russell syndrome and hypomethylation of the H19-DMR. J Hum Genet 2008; 53: 950-955.

24 Bailey W, Popovich B, Jones KL: Monozygotic twins discordant for the Russell-Silver syndrome. Am J Med Genet 1995; 58: 101-105.

25 Samn M, Lewis K, Blumberg B: Monozygotic twins discordant for the Russell-Silver syndrome. Am J Med Genet 1990; 37: 543-545.

26 Schoherr N, Jager S, Ranke MB, Wollmann HA, Binder G, Eggermann T: No evidence for isolated imprinting mutations in the PEG1/MEST locus in Silver-Russell patients. Eur J Med Genet 2008; 51: 322-324. 
27 Gomes MV, Huber J, Ferriani RA, Amaral Neto AM, Ramos ES: Abnormal methylation at the KvDMR1 imprinting control region in clinically normal children conceived by assisted reproductive technologies. Mol Hum Reprod 2009; 15: 471-477.

28 Braulke T: Type-2 IGF receptor: a multi-ligand binding protein. Horm Metab Res 1999; 31: 242-246.

29 Ludwig T, Eggenschwiler J, Fisher P, D'Ercole AJ, Davenport ML, Efstratiadis A: Mouse mutants lacking the type 2 IGF receptor (IGF2R) are rescued from perinatal lethality in Igf2 and Igf1r null backgrounds. Dev Biol 1996; 177: 517-535.

30 Wang ZQ, Fung MR, Barlow DP, Wagner EF: Regulation of embryonic growth and lysosomal targeting by the imprinted Igf2/Mpr gene. Nature 1994. 372: 464-467.

31 Lau MM, Stewart CE, Liu Z, Bhatt H, Rotwein P, Stewart CL: Loss of the imprinted IGF2/cation-independent mannose 6-phosphate receptor results in fetal overgrowth and perinatal lethality. Genes Dev 1994; 8: 2953-2963.
$32 \mathrm{Xu} \mathrm{YQ}$, Grundy P, Polychronakos C: Aberrant imprinting of the insulin-like growth factor II receptor gene in Wilms' tumor. Oncogene 1997; 14: 1041-1046.

33 Yotova IY, Vlatkovic IM, Pauler FM et al: Identification of the human homolog of the imprinted mouse Air non-coding RNA. Genomics 2008; 92: 464-473.

34 Young LE, Fernandes K, McEvoy TG et al: Epigenetic change in IGF2R is associated with fetal overgrowth after sheep embryo culture. Nat Genet 2001; 27 153-154.

35 Gicquel C, Weiss J, Amiel J, Gaston V, Le Bouc Y, Scott CD: Epigenetic abnormalities of the mannose-6-phosphate/IGF2 receptor gene are uncommon in human overgrowth syndromes. J Med Genet 2004; 41: e4.

36 Azzi S, Rossignol S, Steunou V et al: Multilocus methylation analysis in a large cohort of 11p15-related foetal growth disorders (Russell Silver and Beckwith Wiedemann syndromes) reveals simultaneous loss of methylation at paternal and maternal imprinted loci. Hum Mol Genet 2009; 18: 4724-4733. 